\title{
Correction to: Transcranial Direct Current Stimulation in Neuropsychiatric Disorders
}

\author{
André R. Brunoni, Michael A. Nitsche, \\ and Colleen K. Loo
}

\section{Correction to: A. Brunoni et al. (eds.), Transcranial Direct Current Stimulation in Neuropsychiatric Disorders, https://doi.org/10.1007/978-3-030-76136-3}

The original version of chapter 3 , page 29 , chapter 15 , page 283 , chapter 35 , page 667 and chapter 38 , page 729 had wrongly attributed author affiliation to "University of Sao Paulo, Institute of Psychiatry, Sao Paulo, Brazil, Department of Psychiatry University of British Columbia, Vancouver, Canada, Department of Experimental Psychology, University of Oxford, Oxford, UK, Department of Neurology, University Medical Hospital Bergmannsheil, Ruhr-University Bochum, Bochum, Germany, Department of Psychology and Neurosciences, Leibniz Research Centre for Working Environment and Human Factors, Dortmund, Germany".

It has now been corrected to

\section{MA Salehinejad - Chapters 15 and 35}

1 - Department of Psychology and Neurosciences, Leibniz Research Centre for Working Environment and Human Factors, Dortmund, Germany

\section{Vicario - Chapters 15 and 35}

2 - Department of Cognitive Sciences, University of Messina, Messina, Italy

F Vila-Rodriguez - Chapter 15

3 - Department of Psychiatry, University of British Columbia, Vancouver, BC, Canada

R Cohen Kadosh - Chapter 15

4 - Department of Experimental Psychology, University of Oxford, Oxford, UK

S Nikolin - Chapter 35

5 - Black Dog Institute \& School of Psychiatry, University of New South Wales, Sydney, Australia MA Nitsche - Chapters 3, 15, 35 and 38

1 - Department of Psychology and Neurosciences, Leibniz Research Centre for Working Environment and Human Factors, Dortmund, Germany

6 - Department of Neurology, University Medical Hospital Bergmannsheil, Bochum, Germany

The updated online versions of the chapters can be found at https://doi.org/10.1007/978-3-030-76136-3 https://doi.org/10.1007/978-3-030-76136-3_3 https://doi.org/10.1007/978-3-030-76136-3_15 https://doi.org/10.1007/978-3-030-76136-3_35 https://doi.org/10.1007/978-3-030-76136-3_38 